\title{
La mujer ortopedista
}

\author{
The orthopedist woman
}

\author{
Mariano Fernández Fairen, * Marlene Vanessa Salcido Reyna ${ }^{\ddagger}$ \\ *Universidad Internacional de Cataluña, Barcelona, España; ${ }^{\ddagger}$ Coordinación de Enseñanza Red I ISSSTECALI, Mexicali, B.C. México.
}

\begin{abstract}
Resumen
El desarrollo de la mujer dentro de la ortopedia, incluso dentro de la medicina, ha sido complejo, como muchas otras actividades, parecían limitadas por la fuerza; sin embargo, esto carece de sustento científico, el tamaño de la mano no es correlativo con la fuerza del individuo o con la destreza manual. La traumatóloga Susana Alonso Güemes, adjunta del Hospital Ramón y Cajal de Madrid, con 20 años de trabajo de especialidad refiere: «La traumatología es cuestión de técnica, habilidad e inteligencia». La inclusión de la mujer al mundo de la ortopedia parece ser relativamente nueva, la primera mujer ortopedista fue Maud Forrester Brown de Inglaterra en 1924; sin embargo, en México fue hasta 1961 que la Dra. Hayde Esparza Guevara cursó la especialidad y fue reconocida oficialmente en 1974. Más allá de un panorama sombrío, se puede observar un panorama evolutivo, de cambio, donde la mujer se incorporara paulatinamente. La primera razón para escoger la carrera de ortopedia en una encuesta realizada por la organización Ruth Jackson Orthopaedic Society señala que es el gusto por la combinación de la actividad intelectual y manual, así como la satisfacción profesional. Los argumentos más frecuentemente invocados para no optar por ella es la percepción de no encontrar un equilibrio entre el trabajo y la vida privada. Las ortopedistas son mujeres normales con todas las inquietudes y anhelos de todos los seres humanos en general y del conjunto de las mujeres en particular y sobre todo tienen total capacidad de elegir, cursar y desarrollar una carrera profesional como la cirugía ortopédica.
\end{abstract}

Palabras clave: Mujer, ortopedia, liderazgo.

\section{Abstract}

The development of women in orthopedic surgery even in medicine has been complex, as many other activities seemed limited by force, however this lacks scientific support, the size of the hand is not correlated with the strength of the individual or with manual dexterity. Traumatologist Susana Alonso Güemes attached to the Ramon y Cajal Hospital in Madrid, with 20 years of specialty work, refers, "Traumatology is a matter of technique, skill and intelligence». The inclusion of women in the world of orthopedics seems to be relatively new, the first female orthopedist was Maud Forrester Brown in England in 1924, however in Mexico it was until 1961 that Dr Hayde Esparza Guevara studied the specialty and was officially recognized in 1974. Beyond a gloomy panorama, an evolutionary panorama of change can be observed, where women are gradually incorporated. The first reason for choosing an orthopedic career in a survey conducted by the Ruth Jackson Orthopedic Society Organization indicates that it is a taste for the combination of intellectual and manual activity, as well as professional satisfaction. The most frequent arguments invoked for not opting for it is the perception of not finding a balance between work and private life. Orthopedists are normal women with all the concerns and desires of all human beings in general and of all women in particular and above all they have the total capacity to choose, pursue and develop a professional career such as orthopedic surgery.

Keywords: Women, orthopedic, leadership.

\section{Introducción}

Lo que sigue es pasado, presente y, espero, futuro, es una suma de datos y hechos objetivos y una serie de pensamientos y sentimientos subjetivos. He intentado que la subjetividad de mis reflexiones no altere la objetividad tozuda de los acontecimientos. Pero la pureza absoluta es inalcanzable y las historias y la historia, se ven siempre más o menos teñidas por la postura personal que, en mi caso concreto, es claramente pro, como va a verse a continuación. Nunca he tenido duda de la superioridad de la mujer

Correspondencia:

Mariano Fernández Fairen

E-mail: mfernandezfa@uic.es / mferfai@gmail.com

Recibido: 17-11-2021. Aceptado: 17-11-2021.

Citar como: Fernández FM, Salcido RMV. La mujer ortopedista. Orthotips. 2022; 18 (1): 53-63. https://dx.doi.org/10.35366/103733 
como categoría y de su igualdad con el hombre como individuos, siempre que he podido he trabajado con magníficas ortopedistas que me han confirmado lo cabal de mi impresión, con estos prolegómenos vamos al tema que nos ocupa, sabiendo que al tratar en este artículo exclusivamente la problemática de la mujer ortopedista y haber excluido voluntariamente de él los géneros no binarios, de complicadísimo tratamiento, la referencia a sexos en vez de géneros nos parece más adecuada y ajustada y así se ha hecho.

\section{La mujer y la fuerza}

Mi alumna Sara $X$ me dice: "Quiero ser ortopedista, pero no tengo fuerza suficiente...», ¿debe renunciar Sara, mujer inteligente y bien dotada para la cirugía, a seguir su vocación en función de ese parámetro? ¿Hay algún sustento científico de ese aserto o es simplemente un supuesto mítico, repetido y mantenido en el tiempo, que inhabilita a la mujer, sexo débil por naturaleza, para acceder a una carrera profesional de hombres? La visión del ortopedista hercúleo, que trabaja derrochando fuerza en una especie de carpintería esforzada con martillos, sierras y taladros, viene de lejos e, incluso, se ha intentado darle un cierto sustento científico. En ese sentido, Fox y colaboradores se entretuvieron en comparar las manos de gorilas con las de cirujanos ortopédicos y cirujanos generales, tanto staff como residentes, hombres y mujeres. Las tallas de las manos de los ortopedistas en todos los casos eran superiores a las de los cirujanos generales. Pero ¿es esto indicativo de nuestra mayor proximidad filogenética con los gorilas o de nuestra mayor fuerza manual respecto a los cirujanos generales? En lo que se refiere a las ortopedistas, en todos los casos exhibían manos menores que los cirujanos varones, fueran ortopedistas o cirujanos generales, ${ }^{1}$ pero ¿eso significa algo? El tamaño de la mano no es correlativo a la fuerza del individuo, siendo aventurado y anecdótico relacionar ese dato con la capacidad de ejercer la cirugía ortopédica. La traumatóloga Susana Alonso Güemes, médica adjunta del Hospital Ramón y Cajal de Madrid, con 20 años de trabajo en la especialidad, pone perfectamente de manifiesto en sus declaraciones a EFE Salud, en 2019, lo improcedente del mito: «Al principio chocaba porque era un mundo de hombres al que llegaba una chica con la expectativa de ser traumatóloga y, claro, tuve que aguantar comentarios como si me iba a apuntar a un gimnasio para coger fuerza, pero no me ha hecho falta la fuerza porque la traumatología es cuestión de técnica, habilidad e inteligencia. Si hay que recurrir a la fuerza es porque algo no se hace bien. La fuerza física nunca ha sido un hándicap para mí». ${ }^{2}$ Idéntica es la experiencia aportada por Laura Muñoz, cirujana ortopédica y traumatóloga del Servicio de Cirugía Infantil del Hospital General Universitario «Gregorio Marañón» de Madrid: "La traumatología no es cosa de fuerza». ${ }^{3}$

Queda pues claro qué le he dicho a Sara: sigue el camino que has elegido, que hará plena tu vida.

\section{Algo de historia}

Pero evidentemente mujer ortopedista lleva en el enunciado «mujer» antes que «ortopedista», y ser mujer, ortopedista o cualquier otra cosa, no es fácil, tal como ironiza Carmen Rico-Godoy en el título de su libro Cómo ser una mujer y no morir en el intento, ${ }^{4}$ incluso en esta era de avances intelectuales y sociales promovidos por el feminismo y la posición racional de muchos hombres. En EUA hay que remontarse a 1846 para encontrar la primera mujer graduada en Medicina, la Dra. Elizabeth Blackwell, nacida en Inglaterra en 1821 (Figura 1); en España, Dolores Aleu Riera se convirtió en la primera mujer licenciada y doctora en Medicina en 1882; y en México, Matilde Montoya Lafragua fue la primera mujer en obtener ese título en el año 1887, ejerciendo como médico

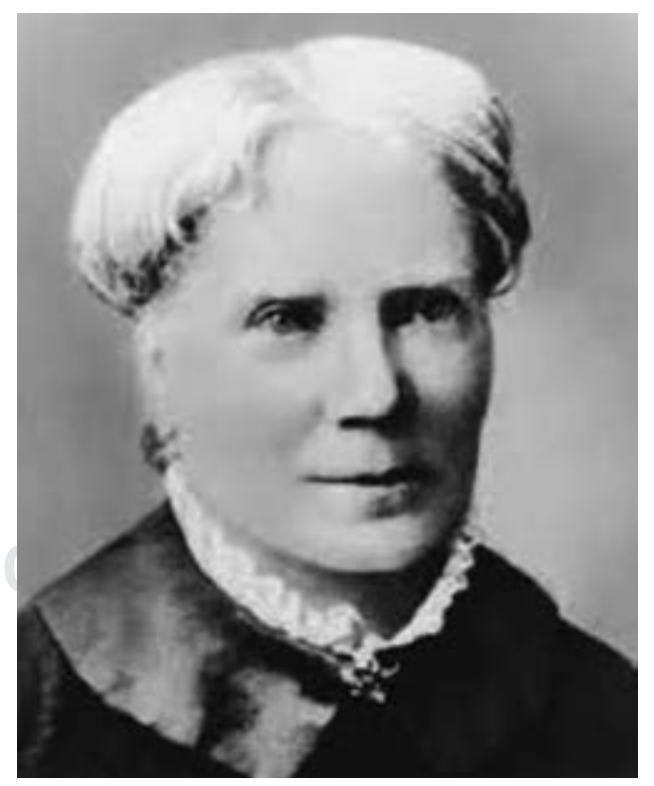

Figura 1: Dra. Elizabeth Blackwell. Primera mujer de EUA graduada en Medicina en 1846. 

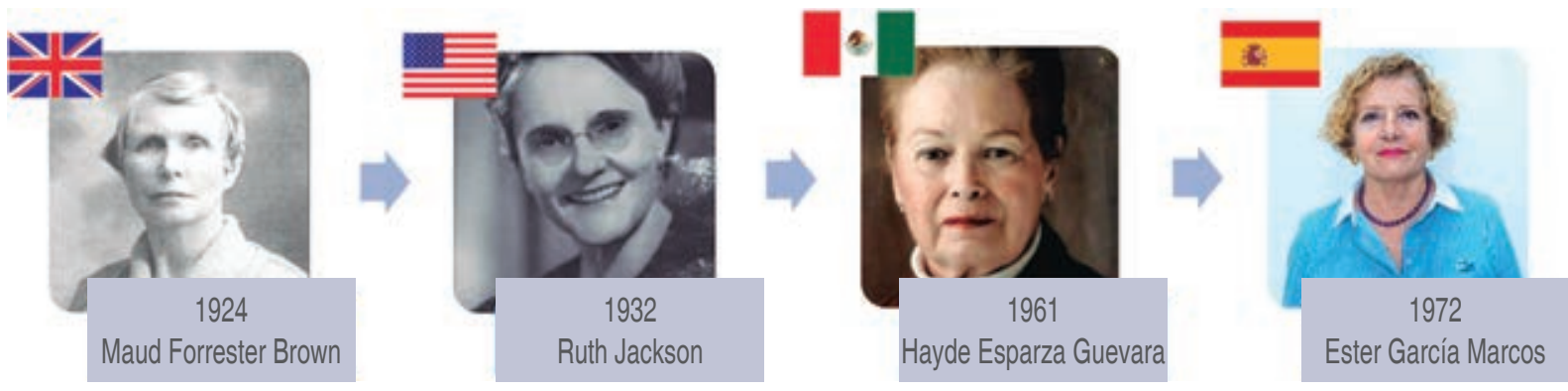

Figura 2: Evolución cronológica de la mujer ortopedista a nivel mundial.

cirujano y obstetra. ${ }^{5}$ De cualquier manera, no hay que retroceder más allá de medio siglo para que las mujeres graduadas en Medicina fueran encaminadas a especialidades consideradas más femeninas, como Pediatría, Ginecología, Medicina de Familia, Dermatología, Oftalmología, etcétera. En Inglaterra, Maud Forrester Brown fue la primera mujer en practicar la ortopedia en 1924. En EUA lo fue Ruth Jackson, quien al terminar la residencia abrió su oficina de ortopedista en lowa en 1932, siendo certificada por el American Board of Orthopaedic Surgeryen 1937. ${ }^{6}$ En España, la primera mujer en ocupar una plaza de especialista en Cirugía Ortopédica y Traumatología (COT) fue Ester García Marcos en 1972, en el Hospital Insular de Las Palmas de Gran Canaria. No tuvo la misma suerte otra pionera española como Consuelo García Blanque, de Granada, que se tituló como especialista en Traumatología, Cirugía Ortopédica y Cirugía General en 1979, pero que, viviendo en Barcelona, no consiguió plaza de traumatólogo hasta mediados de los años 80 por ser mujer y no ser catalana. ${ }^{7}$ En México, la Dra. Hayde Esparza Guevara cursó la residencia en la especialidad de Ortopedia y Traumatología en el Hospital «Rubén Leñero» del Departamento del Distrito Federal a partir de mayo de 1961 y fue en 1974 cuando el Consejo Mexicano de Ortopedia le entregó su Certificado de Reconocimiento, siendo la primera mujer ortopedista certificada en el país (Figura 2). ${ }^{8}$

La posición masculina, o al menos la de los mentores encargados de llevar a buen puerto las vocaciones ortopédicas, era cuando menos negativa u opuesta a la entrada de la mujer en ese terreno, lo que le dijo a la arriba mencionada Consuelo García el catedrático al que fue a tocar a su puerta para devenir ortopedista, que «no es profesión para mujeres», era sentir general tal como resulta obvio de la queja de un cirujano del Hospital «Magdalena de las Salinas» al jefe de residentes por enviar a la Dra. Fryda Medina Rodríguez



Figura 3: Dra. Fryda Medina Rodríguez, distinción otorgada por la Asociación Nacional de Mujeres Médicas «Dra. Matilde P. Montoya».

al haberle solicitado un residente de Traumatología: «te pedí un residente, no una vieja». ${ }^{9}$ La «vieja» ha llegado a ser Médica del Año 2019, distinción otorgada por la Asociación Nacional de Mujeres Médicas «Dra. Matilde P. Montoya» (Figura 3).

Este panorama se ha ido revirtiendo poco a poco, abriéndose paulatinamente el acceso e incorporación de la mujer a la COT tal como va a verse, aunque eso no es en absoluto general, en 2019, Estonia encabezaba la lista de países con mayor número, con $26.4 \%$ de mujeres ortopedistas, y España a continuación con 25\%, ${ }^{10,11}$ en el otro extremo se encuentran Laos y Camboya con $0 \%$ de ortopedistas femeninas (Tabla 1). ${ }^{10}$

\section{Los números cantan}

En 1960 la mujer constituía 6\% de la población médica (índice de distribución; el valor $50 \%$ para cada sexo corresponde a la igualdad de distribución por sexos), pasando a ser $30 \%$ de la misma en el año $2000,{ }^{12}$ y $44 \%$ en $2014,{ }^{13}$ hoy día en España más de $50 \%$ de los estudiantes de Medicina son mujeres, en una relación similar a la de México y 
Estados Unidos, en neto avance respecto a $30 \%$ del año 2000 , y a aproximadamente $20 \%$ español desde la década de los 70 .

En España, en 2017, la COT estaba copada fundamentalmente por hombres al constituir $80 \%$ de los efectivos de la especialidad, ${ }^{14,15}$ aunque en la encuesta realizada por EFORT en 2019 el porcentaje de españolas en COT alcanzaba $25 \% .{ }^{11}$ Es la especialidad más alejada de la paridad mujer-hombre, ${ }^{15}$ por esto y por otras razones como que los puestos y cargos relevantes siguen ocupados por varones, como por ejemplo $90 \%$ de los docentes e investigadores en la universidad pública española, ${ }^{15}$ la plena visibilidad de la mujer en COT tardará todavía algún tiempo. ${ }^{16}$ En ese sentido, en España, a nivel de los residentes que comienzan la especialidad, existe ya esa paridad entre ambos sexos, ${ }^{16}$ igual que sucede en los estudios de Medicina, y hay instituciones como por ejemplo el Hospital Universitario Virgen Macarena de Sevilla en la que las mujeres ortopedistas representan la tercera parte de los ortopedistas del centro. ${ }^{17}$

En México, en 2013, las mujeres suponían 17\% de los residentes en COT. ${ }^{5}$ En 2018, 93\% de los especialistas mexicanos en COT eran hombres. ${ }^{18}$ En tanto $53 \%$ de ortopedistas mujeres tenían su certificación vi- gente, ese porcentaje era de $48 \%$ entre los hombres. ${ }^{18}$ En 2021, las mujeres siguen representando menos de $10 \%$ de los ortopedistas del país. La diferencia en la cantidad global de especialistas masculinos y femeninos es abismal, pues ellas son unas $600 \mathrm{y}$ ellos aproximadamente 8 mil. ${ }^{9}$ Algo parecido sucede en Canadá donde, en 2018, las mujeres eran $11 \%$ de los cirujanos ortopédicos y $18 \%$ de los miembros de la Canadian Orthopaedic Association (COA). ${ }^{19}$

En EUA, en el año académico 2008-2009, 13\% de los residentes de COT, $4 \%$ de los cirujanos ortopédicos certificados, $5 \%$ de los profesores titulares, $12 \%$ de los profesores asociados, $16 \%$ de los ayudantes y $23 \%$ de ayudantes fueron mujeres. ${ }^{20}$ Sin embargo, la proporción de mujeres residentes en COT aumentó cada año, habiéndolo hecho en un $27 \%$, de 11 a $14 \%$, entre 2005 y 2017 , y las enseñantes de COT a tiempo completo supusieron $18 \%$ del cuerpo docente de las escuelas de Medicina estadounidenses en el curso 2016-2017. ${ }^{21}$ En $2019,6 \%$ de especialistas certificados miembros de la American Academy of Orthopaedic Surgeons (AAOS) y $15.4 \%$ de residentes eran mujeres. ${ }^{11}$ Todos los porcentajes de presencia femenina en COT son los menores cuando se comparan con el resto de las especialidades médicas. ${ }^{21-25}$ Proyectando

Tabla 1: Diversidad de mujeres ortopedistas a nivel mundial.

\begin{tabular}{|c|c|c|c|c|c|c|}
\hline \multirow[b]{2}{*}{ Lugar } & \multirow[b]{2}{*}{ País } & \multirow[b]{2}{*}{ Población (millones) } & \multirow[b]{2}{*}{ Cirujanos ortopédicos } & \multirow[b]{2}{*}{ Cirujanos: población } & \multicolumn{2}{|c|}{ Mujeres cirujanas ortopédicas } \\
\hline & & & & & $\mathrm{n}$ & $\%$ \\
\hline 1 & Estonia & 1.3 & 110 & $1: 12,045$ & 29 & 26.4 \\
\hline 2 & Suecia & 10.1 & 1,376 & $1: 7,316$ & 231 & 16.8 \\
\hline 3 & Brunéi & 0.4 & 15 & $1: 28,666$ & 2 & 13.3 \\
\hline 4 & Canadá & 37.6 & 1,659 & $1: 22,658$ & 199 & 12.0 \\
\hline 5 & Colombia & 50.3 & 2,020 & $1: 24,900$ & 208 & 10.3 \\
\hline 6 & Malasia & 32.6 & 982 & $1: 33,197$ & 98 & 10.0 \\
\hline 7 & Hong Kong & 7.4 & 470 & $1: 15,744$ & 38 & 8.1 \\
\hline 8 & Tanzania & 50.0 & 118 & $1: 423,728$ & 9 & 7.6 \\
\hline 9 & Francia & 67.0 & 3,503 & $1: 19,126$ & 248 & 7.1 \\
\hline 10 & Chile & 18.9 & 794 & $1: 23,803$ & 49 & 6.2 \\
\hline 11 & Estados Unidos & 329.1 & 27,651 & $1: 11,900$ & 1,673 & 6.1 \\
\hline 12 & Indonesia & 270.6 & 1,000 & $1: 252,897$ & 54 & 5.4 \\
\hline 13 & Nueva Zelanda & 4.8 & 302 & $1: 15,894$ & 15 & 5.0 \\
\hline 14 & Japón & 126.7 & 21,275 & $1: 5,955$ & 1,040 & 4.9 \\
\hline 15 & Reino Unido & 66.9 & 2,960 & $1: 22,591$ & 141 & 4.8 \\
\hline 16 & Australia & 25.4 & 1,334 & $1: 19,538$ & 57 & 4.3 \\
\hline 17 & Kosovo & 1.8 & 78 & $1: 23,076$ & 3 & 3.8 \\
\hline 18 & Tailandia & 69.6 & 2,430 & $1: 28,641$ & 92 & 3.8 \\
\hline
\end{tabular}


en el tiempo la evolución de los porcentajes de residentes femeninas en COT hasta 2019, no será hasta aproximadamente 2060 cuando las mujeres supongan un $30 \%$ de esa condición. ${ }^{24}$ En el año 2134 podría alcanzarse la paridad de residentes femeninas en COT y en el resto de las especialidades médicas y en 2155 la equivalencia de su porcentaje con la población femenina norteamericana total (50.8\% de población femenina). ${ }^{25}$ Para conseguir un $36.3 \%$ de mujeres en COT, lo que resultaría la paridad con la profesión médica en general, habrá que esperar a 2236, en tanto que la paridad con la población general EUA ( $50.8 \%$ de mujeres) podría lograrse en $2354 .^{26}$

\section{Ser o no ser}

En una encuesta realizada entre las afiliadas a la Ruth Jackson Orthopaedic Society (RJOS), organización independiente fundada en EUA en 1983, que reúne a las mujeres relacionadas con la COT y a la que pertenecen $42 \%$ de las miembros de la American Association of Orthopaedic Surgeons, se les inquirió por qué habían elegido ser o no ser ortopedistas, como razones más comunes para una elección afirmativa citaron el disfrutar con la combinación de actividad intelectual y trabajo manual $(71 \%)$, la satisfacción profesional (54\%) y el estímulo intelectual (53\%) que representa esta disciplina. Los argumentos más frecuentemente invocados para no optar por ella incluyeron la percepción de no poder alcanzar un buen equilibrio entre el trabajo y la vida privada $(78 \%)$, el sentimiento de que se requiere demasiada fuerza física (74\%) y la falta de tutela sólida en esa orientación en la escuela de medicina o incluso antes (69\%). ${ }^{23}$ Estos argumentos disuasorios eran similares a los expresados por estudiantes de medicina ante la eventualidad de seguir o no una carrera quirúrgica en una encuesta de la Harvard Medical School publicada en 2021, las muchas horas de trabajo profesional y las pocas para otras actividades fueron el mayor impedimento referido por ambos sexos. Más mujeres que hombres manifestaron preocupaciones sobre el tiempo para casarse $(p=0.042)$, el tiempo a pasar con la familia $(p=0.015)$, y tiempo para tener un hijo ( $p<0.0001)$, tomar permiso por maternidad/ paternidad durante la residencia $(p<0.0001)$ y ser demasiado mayor después de la residencia para tener un hijo $(p<0.0001) .^{27}$

Entre las influencias positivas que las condujeron a la COT se cuentan tener un miembro de la familia ortopedista, haber pasado la interesada o alguien de su familia por una experiencia ortopédica, una historia personal de interés hacia el deporte, la influencia de los mentores o el acceso al aparato locomotor en la escuela de medicina, y la seducción de una especialidad tan amplia y variada, quirúrgica o no. ${ }^{23}$ Esta atracción es tan importante como para hacer que en 2016 en números brutos, no porcentuales, y exceptuando cirugía general, la cirugía ortopédica sea la especialidad quirúrgica en la que hay más residentes femeninos..$^{28}$ Como disuasores se reportaban el desagrado hacia el trabajo manual y el quirófano, no haber tenido contacto con la problemática ortopédica o no haberse interesado por ella en la escuela de medicina, o haber sufrido alguna experiencia desagradable en el campo de la COT o con algún cirujano ortopédico. ${ }^{23} \mathrm{En}$ otra encuesta entre residentes realizada por la AAOS en $2011,12 \%$ de encuestadas señalaban que para ellas había sido una barrera la mala aceptación de la mujer por parte de los ortopedistas senior. En sentido positivo, $59 \%$ de mujeres y $25 \%$ de hombres indicaron que tener un modelo a seguir de su mismo sexo fue un factor positivo para elegir la cirugía ortopédica, ${ }^{29}$ pero eso, como va a verse, presenta problemas en la cotidianidad y en ese papel de modelo no hay por qué contar con que sea del mismo sexo que el discente. ${ }^{28}$

Más de la mitad de las mujeres encuestadas señalaron la escasa o nula participación femenina entre los docentes de su programa de residencia. ${ }^{23}$ Se ha postulado que la contribución de mujeres en la docencia correspondiente aparece como un factor de la distribución desigual de mujeres residentes en ortopedia en los Estados Unidos. ${ }^{20}$ La presencia de profesoras en las escuelas de Medicina se ha relacionado con la diferente elección de las especialidades que las estudiantes eligen la cirugía como carrera, concordando esto con trabajos que sugieren que la ausencia de una "masa crítica» de mujeres puede disuadir a las solicitantes de elegir un campo o un programa de residencia determinados. ${ }^{20}$ Revisando los programas de residencia en COT listados en la American Medical Association (AMA), los que congregaban más mujeres residentes tenían más mujeres en el profesorado $(p=0.001)$, mayor porcentaje de profesores que eran mujeres $(p<0.001)$, más profesoras asociadas $(p<0.001)$, más mujeres en puestos de liderazgo $(p<0.001) .{ }^{30}$ Una vez que la proporción de profesoras supere $30-35 \%$ del profesorado total, su impacto en el cambio cultural será evidente, tal como predice la teoría de la masa crítica aplicada a la equidad de sexo en instituciones académicas sanitarias. ${ }^{31}$ 
Hay que tener presente que el porcentaje de mujeres que opta por la residencia en COT, en EUA, es el más bajo entre los residentes de todas las especialidades médicas, ${ }^{32}$ lo que hace cuestionarse el interés de la mujer por la ortopedia, ${ }^{33}$ por el contrario, en EUA entre 2010 y 2014, el éxito de las aspirantes a la especialización en COT fue mayor que el de los varones (96 vs $81 \%$; $p<0.001$ ), esto fue así teniendo en cuenta que dentro de COT la concurrencia femenina a ortopedia pediátrica fue la mayor con un $25 \%$, seguida de la cirugía de pie y tobillo con $14 \%$, siendo la menos pretendida la cirugía de raquis con un $3 \%,{ }^{34}$ en cirugía ocurre algo similar si la mujer es soltera pero esa ventaja se invierte si es casada, y decae aún más si tiene hijos. ${ }^{35}$

\section{Resabios sociales}

Antes de comenzar ya hay «pegas» y discriminación, tal como señalan Flores-Domínguez y colaboradores, en el entorno familiar de la futura profesional, "los padres de familia, incluso las madres, son renuentes a que sus hijas estudien medicina porque quieren que "pueda tener una familia", mientras se regodean al saber que su hijo varón quiere ser médico". ${ }^{36} \mathrm{En}$ el caso concreto de COT, considerada como un «club de chicos", no se puede ser cirujano ortopédico y madre. ${ }^{10}$ Las actitudes negativas influyen de manera importante en la elección de la especialidad. En 2009, en Reino Unido, $42 \%$ de los ortopedistas varones estuvieron expuestos a actitudes negativas respecto a las ortopedistas femeninas, pero ese porcentaje era significativamente mayor en las mujeres que consideraban la COT como una posible carrera, de tal forma que $62 \%$ de las mismas renunció a emprenderla. ${ }^{37} \mathrm{En}$ la encuesta ya citada de la Harvard Medical School $69 \%$ de todos los estudiantes y $75 \%$ de los que se decidieron por la cirugía reportaron haber sido desanimados verbalmente en cuanto a seguir esa carrera. Las mujeres percibieron más comentarios desmotivadores por su condición de mujer $(p<0.0001)$, edad $(p<0.0001)$ y aspiraciones familiares $(p=0.043),{ }^{27}$ y eso a lo largo de toda su carrera; en una encuesta realizada en 2013 entre las docentes médicas de la Universidad de Kansas reportaban haber sido tratadas con «prejuicios tácitos», «ser ignoradas» y ser vistas y tratadas como "otras", las involucradas relataron haber afrontado esas situaciones restándoles importancia, manteniendo la distancia, empleando el humor y usando símbolos genéricos comunes como la bata blanca en el desempeño de sus funciones. Explicaron la consecución del éxito en esa tarea uniendo inteligencia, meritocracia, ecuanimidad y una salvaguardia cuidadosa de su feminidad. ${ }^{38}$

Una vez en vías de sobrepasar los prejuicios históricos clásicos comentados antes, y esto en COT no debe haberse logrado del todo, ya que sólo $0.92 \%$ del total de residentes son féminas que eligen esta especialidad, ${ }^{21}$ las ortopedistas detectan en su actividad más «micromachismos» sociales que en el propio entorno médico. En efecto, con frecuencia, el paciente y/o sus allegados, incluso paradójicamente aún más si son mujeres, buscan en el contacto con el médico la figura masculina del «Doctor» para otorgarle credibilidad y confianza. Si es una mujer la que los atiende, por más que sea su nivel personal y profesional, no pasa de ser considerada frecuentemente como alguien auxiliar o, a lo sumo, en formación, especialmente si es joven o su aspecto lo es, reduciéndola al papel secundario que la historia y la costumbre le ha otorgado en estos temas, de todas formas eso está cambiando poco a poco, en 2009, en Reino Unido, $89 \%$ de pacientes no expresaban preferencia porque su ortopedista fuera de uno u otro sexo, $75 \%$ decían creer que las ortopedistas eran tan diestras como los ortopedistas, e incluso un 4\% afirmaban preferir una mujer ortopedista a un hombre. ${ }^{37}$ En 2019, en una encuesta de la Universidad de Carolina del Norte, 84\% de los pacientes no tenía preferencias a la hora de ser tratados, $14.5 \%$ preferían una ortopedista y $1.6 \%$ un ortopedista. Un tercio de los pacientes encontraban importante la apariencia física, sexo, raza y edad, $27 \%$ los años de práctica y $16.7 \%$ la reputación y prestigio del especialista. ${ }^{39}$

La mujer tiene comúnmente salarios más bajos, menores ayudas económicas a su actividad científica, menos oportunidades para exponer y publicar esa actividad, y su promoción es menor, más costosa y lenta, ${ }^{40}$ la diferencia entre sexos ya citada de ocupación de los cargos jerárquicamente más altos con mayores percepciones económicas, la menor realización de trabajo nocturno y guardias mejor pagadas, la menor vida laboral por bajas y permisos por motivos familiares que dificultan su carrera profesional y disminuyen sus derechos laborales pueden ser motivos de esa menor retribución de la mujer durante su carrera y en su jubilación, pero incluso trabajando las mismas horas hay una diferencia significativa entre los emolumentos de unas y otros $(p<0.001)$, debido a que la mujer realiza un menor volumen de casos en el mismo número de horas. Entre los ortopedistas hombres o mujeres que efectuaban más de 26 pro- 
cedimientos al mes no había diferencia, también eran comparables los ingresos en aquéllos que llevaban más de 20 años de práctica. ${ }^{41}$

Ya se ha dicho antes que la visibilidad de la mujer como especialista formado y responsable no se ha logrado plenamente todavía, de forma similar a lo que ocurre en otras parcelas, la ratio de ocupación de posiciones preminentes dentro de la profesión es favorable a los varones, con lo que eso tiene de equívoco de cara a la consideración social de las especialistas, en EUA, en el curso académico 20152016, de un cuerpo docente en COT constituido por $18 \%$ de mujeres, éstas ocupaban sobre todo plazas de rango inferior y sólo $8.7 \%$ de las plazas de profesor y $1 \%$ de las cátedras, con una evidente falta de proporcionalidad respecto a los varones, ${ }^{21}$ en otro estudio realizado también en EUA, en 2013, 10\% de las docentes en COT eran instructores, $56 \%$ ayudantes, $22 \%$ profesores asociados y $9 \%$ profesores titulares. ${ }^{42}$

De todas formas ese argumento, atractivo a la hora de buscar explicaciones a la infraconsideración de las mujeres ortopedistas, no es general y si bien es cierto que globalmente predominan los hombres en los puestos relevantes, la diferencia va atenuándose con el tiempo en muchos casos, comparar estos últimos datos con los correspondientes a los mismos conceptos en el curso 2008-2009 explicitados antes y en otros desaparece cuando esa comparación se hace atendiendo a los porcentajes alcanzados por cada sexo en cada categoría dentro del global de individuos (índice de concentración; la igualdad de porcentaje corresponde a la igualdad entre sexos). Como ejemplo, en Canadá la presencia de mujeres ortopedistas en comités, presidencia y moderación de sesiones, y presencia en el podio de las reuniones de la COA fue en 2018 equivalente a su participación porcentual como miembros de dicha sociedad. ${ }^{19}$

Hay muchos más ejemplos de lo que se está diciendo. Los imperativos sociales, junto a la reserva propia de la mujer y quizás la aceptación inconsciente de una supuesta minusvalía, admitida de inmediato por el varón, encantado con esa situación, se pueden apreciar en múltiples facetas. Entre 2006 y 2017, en los artículos publicados en Clinical Orthopaedics and Related Research (CORR), Journal of Bone and Joint Surgery, American volume (JBJS) y American Journal of Sports Medicine (AJSM), una mujer aparecía como primer autor en $13 \%$ y en $10 \%$ en último lugar. El incremento de mujeres como primer autor entre 2006 y 2017 fue de 11 a 17\%, significativo pero claramente insuficiente. 43
Un completísimo estudio bibliométrico de la literatura ortopédica en inglés de los últimos 30 años muestra cómo la mujer firmaba como primer autor en un $11 \%$ y aparecía como autor corresponsal en 9\% de artículos publicados en 1985-1987, tanto en un caso como en otro, esos artículos tenían más autores, más instituciones, países, referencias y páginas, en trabajos de un solo autor la mujer lo era en la mitad de los casos que el hombre, algo se ha enmendado con el paso del tiempo y así en 2015-2016 la figura de la mujer como primer autor ascendió a 24\%, igual que su presencia como editores y en los consejos editoriales de las publicaciones de la especialidad, ${ }^{44}$ en las tres revistas ortopédicas citadas arriba había en 2007 un 3\% de mujeres en sus consejos editoriales y en 2017 un $9 \% .45$

En los congresos o convenciones anuales de la AAOS, entre 2015 y 2019, las mujeres representaron $5.9 \%$ de los moderadores e instructores de cursos, el porcentaje de mujeres moderadoras de sesiones ascendió de $6 \%$ en 2015 a $8.6 \%$ en 2019 , en tanto que el número de instructoras creció de $3.4 \%$ en 2015 a 5.6\% en 2019, ajustando las cifras a la posible presencia dual múltiple de un mismo individuo en uno u otro papel a lo largo del programa científico de las reuniones, $6.7 \%$ de moderadores e instructores fueron mujeres, $6.3 \%$ en 2015 y $7.7 \%$ en 2019.46

Para terminar este punto, cabe citar a los medios de comunicación que, por más que hablen de su incorporación plena a los movimientos "progresistas» de las políticas de género, colaboran intensa y diariamente en la exhibición de la mujer que destaca como algo extraordinario, lejos de lo que debe ser considerado habitual y normal, así, por ejemplo, en la lista que publica anualmente Forbes destacando los 10 mejores especialistas en COT de España, en 2021 no incluye entre ellos ninguna ortopedista. Evidentemente hay que contar con la poderosa, parcial y nada inocente faceta de merchandising que hace que el medio acomode su mensaje a la tendencia social del momento.

\section{Cómo son las ortopedistas}

Son mujeres normales con todas la inquietudes y anhelos de todos los seres humanos en general y del conjunto de las mujeres en particular, y sobre todo tienen total capacidad para elegir, cursar y desarrollar una carrera profesional como lo es la cirugía ortopédica.

En la encuesta de la RJOS mencionada arriba, las especialidades más comunes ostentadas por las 
encuestadas fueron la cirugía de mano (24\%), ortopedia general $(20 \%)$, ortopedia pediátrica $(19 \%)$ y traumatología deportiva (15\%), por detrás quedan la cirugía del pie, la traumatología, la cirugía de cadera y rodilla, los tumores y, por último, el raquis, un $42 \%$ trabajaba en un entorno académico y $21 \%$ estaban empleadas en un hospital, dos tercios dedicaban entre 40 y 70 horas semanales a actividades clínicas asistenciales directas, $52 \%$ tenía hijos, ${ }^{23}$ los resultados de esa encuesta respecto al encuadramiento de las ortopedistas en las diferentes especialidades son similares a los obtenidos de otra encuesta dirigida en 2020 a la RJOS, la Asociación Ortopédica de Texas (TOA) y a una página de Internet para mujeres en ortopedia, que cubría el área de subespecialidades, incluyendo cirujanas ortopédicas en ejercicio, especialistas en formación o residentes. La cirugía de mano ostentaba el primer lugar con $24 \%$ de presencia femenina a continuación, la cirugía pediátrica con $22.6 \%$ y la traumatología deportiva con $16.3 \%,{ }^{47}$ aunque la cirugía ortopédica pediátrica sigue siendo una de las opciones más elegidas por las féminas, las mujeres que se integran en la ortopedia académica optan cada vez más por otras subespecialidades no pediátricas. ${ }^{48}$

Respecto a las ortopedistas docentes, en EUA en 2013, ocupaban todas las subespecialidades de COT, con el número más alto en ortopedia pediátrica, cirugía de mano, trauma y deporte; $93 \%$ estaban satisfechas con su elección de subespecialidad y $77 \%$ volverían a entrar en la docencia otra vez; $73 \%$ tenían marido o pareja estable y $62 \%$ al menos un hijo. ${ }^{42}$

\section{Qué riegos específicos corren las ortopedistas}

La mujer corre riesgos específicos claros en cuanto a la exposición a la radiación debiendo usar siempre las protecciones reglamentarias y una doble capa de plomo durante el embarazo, ${ }^{49}$ respecto al cemento acrílico se ha demostrado que el metacrilato de metilo es feto tóxico a concentraciones > 1,000 ppm pero el uso apropiado de sistemas de casco protector y mezcla al vacío minimiza la exposición al mismo. ${ }^{50}$

Más insidiosos son los efectos de las largas jornadas de trabajo y los turnos nocturnos sobre las ortopedistas embarazadas, en EUA, en las ortopedistas que trabajan más de 60 horas por semana, la probabilidad de parto prematuro es cinco veces más alta que el promedio de mujeres norteamericanas, el riesgo de que se complique el embarazo es mayor en las ortopedistas (31.2\%) que en la población general $(14.5 \%){ }^{51}$

\section{Buscando soluciones}

El problema es tan multifactorial que resulta muy difícil hacerse con él en su totalidad, ${ }^{52}$ interviniendo factores personales, familiares, sociales, culturales, económicos, históricos, convencionales, éticos y religiosos, ${ }^{53}$ y eso repercute en lo inmediato en la búsqueda y puesta en marcha de soluciones.

Hay que hacerse visible, la invisibilidad crónica tradicional de la mujer y la resistencia a romperla ha sido uno de los puntos negativos para la eclosión del papel femenino en cualquier faceta social y profesional, por eso hay muchas mujeres que han optado por escribir, exponer sus ideas, contar sus experiencias y proponer soluciones para ese repudio, sirviendo de modelo a las que ya están y a las que vienen, ${ }^{54}$ de hecho, la bibliografía de este artículo refleja bien a las claras que la mayoría de los autores en este tema son mujeres ortopedistas, que han emprendido ese camino con la preocupación y deseo de enmendar las cosas, el punto negro es la también ostensible escasa participación y cooperación del hombre en ese camino.

En ese mismo sentido, el 11 de enero se celebra el Día Internacional de la Mujer Médico con cuyo motivo los medios de comunicación dan cierta visibilidad a mujeres que laboran en el campo de la salud, pero esto que es digno de mención no debe hacer olvidar que esas mujeres trabajan en eso todo el año y muchas toda su vida, por tanto, todos los días deben ser 11 de enero y no hay que dejar pasar ni una sola oportunidad de estar presente sea el día que sea.

En el campo de las asociaciones hay que señalar y hacer hincapié en aquéllas que velan por la diversidad, considerándola esencial para maximizar la presencia y la acción social de sus integrantes y optimizar su talento y habilidades, las estructuras sociales y las organizaciones que son diversas son dinámicas, atractivas y exhiben una mayor equidad y eficacia en la toma y realización de decisiones. ${ }^{10,11,22}$ Los modelos a seguir variados, los buenos mentores y los patrones de trabajo flexibles son esenciales para conseguir el apoyo y la representación de todos los grupos que participan en un espacio social determinado ${ }^{55}$ y esos tres puntos se revelan fundamentales para la plena participación de la mujer en el campo de la COT. En lo concerniente a ésta, hay que citar la International Orthopaedic Diversity Alliance (IODA), 
The Perry Initiative $y N^{\text {th }}$ Dimensions. IODA nace en 2019 de la colaboración internacional con el objetivo de unir a los líderes en COT y en la industria para promover la diversidad, la equidad y la inclusión, y proporcionar un foro mundial para identificar, desarrollar, compartir, apoyar, participar y promover la diversidad en todas las facetas de la COT, está abierta a todos en el sector de la salud y la membresía es gratuita. ${ }^{56}$ The Perry Initiative fue fundada en 2009 en honor de una magnífica mujer, Jacquelin Perry (1918-2013), quien trabajó incansablemente enseñando e investigando en ortopedia y biomecánica. Su finalidad es inspirar y enseñar a las mujeres jóvenes a ser líderes en los campos de la cirugía ortopédica y la ingeniería, campos en los que las mujeres están drásticamente infrarrepresentadas. ${ }^{57} N^{\text {th }}$ Dimensions fue fundada en 2004 por ortopedistas trabajando en colaboración con instituciones académicas, cirujanos comunitarios y la industria para abordar la escasez de mujeres en cirugía ortopédica. ${ }^{58}$

Otra buena iniciativa, en el camino señalado por Flores-Domínguez y colaboradores ${ }^{36}$ de participación de la mujer en instituciones y órganos colegiados, es la constitución de la Asociación Nacional de Mujeres Ortopedistas (ANMO) en México.

De lo que no cabe la menor duda es que hombres y mujeres han de tener claro que ambos son perfectamente capaces de desarrollar cualquier tipo de actividad, la COT entre ellas, sólo el convencimiento íntimo, racional y universal de esto puede mejorar la situación actual y en cuanto a cómo hacer, en asuntos tan sensibles como la libertad de elección y acción, la igualdad de oportunidades y la leal competencia son las personas las que deben decidirlo y elevarlo a la categoría de absoluto en la vida diaria. Está claro que en el nivel categórico de los sexos se presentan las lógicas, humanas, innegables e inevitables diferencias individuales, sean del sexo que sean, que hay al mismo tiempo que reconocer, conservar y respetar para que el individuo prevalezca por encima de cualquier consideración limitante, pacata e injusta de cualquier tipo de discriminación positiva. Hay que huir de políticas y leyes proteccionistas oportunistas que no hacen más que convertir una situación indeseable en otra igualmente indeseable, reduciendo intencionada y arteramente la valía real de la mujer y el mérito personal a graciosa concesión del gobierno de turno. ¿Son la solución iniciativas como la Ley Orgánica Española 3/2007 para la igualdad efectiva de mujeres y hombres, ${ }^{59}$ que anima a adoptar medidas específicas en favor de las mujeres para corregir situaciones de desigualdad, como hacer preceptivo que ocupen $40 \%$ de los cargos de responsabilidad, y todas las leyes que han venido después?, evidentemente no, ${ }^{15,60,61}$ igual que no lo es colocar en cargos ejecutivos de responsabilidad a incapaces por razones de "cuota», amiguismo, enchufismo, clientelismo o incluso sólo por corrección política, como ha sido el caso de eximias máximas responsables de la sanidad en España y en Cataluña, mientras que sí lo es acceder a esos cargos a través de procesos equitativos y sin restricciones, que hagan valer los auténticos méritos propios, en una sociedad que haya prescindido de los prejuicios y mitos del pasado/presente. Si no se hace así, nos encontraremos que bajo un sistema de promoción profesional aparentemente igualitario, basado en los méritos y la experiencia, subsisten graves limitaciones sustanciales para el desarrollo de una carrera profesional deseada ${ }^{62}$ y esto no es deseable ni recomendable.

\section{Conclusión}

Esta revisión pone de manifiesto la brecha entre mujer y hombre en COT, sus posibles razones, la evolución de la misma en el tiempo y sus potenciales soluciones, hay que reconocer que se ha avanzado en estos últimos años, pero falta todavía mucho para lograr un total, justo y equitativo posicionamiento de la mujer en este campo, trabajemos en ello.

Como conclusión, una reflexión de la Dra. Cristina Asenjo Gismero, traumatóloga y cirujana ortopédica en el Hospital Beata María de Madrid, con motivo del Día Internacional de la Mujer y Especialidades Médicas: "Ser buen traumatólogo no depende del sexo, sino de la pasión y la ilusión que le pongamos a lo que hacemos, como todo en esta vida", con otra de la ya citada Dra. Laura Muñoz: «Nosotras podemos y lo hacemos mejor que muchos hombres. No depende de fuerza sino de habilidad y de nuestra inteligencia. No hay ninguna restricción que nos impida ser traumatólogas. Ni mentales, ni físicas». No puedo estar más de acuerdo con ambas.

\section{Referencias}

1. Fox JS, Bell GR, Sweeney PJ. Are orthopaedic surgeons really gorillas? BMJ. 1990; 301 (6766): 1425-1426. doi: 10.1136/ bmj.301.6766.1425.

2. Soteras A. Mujeres pioneras en especialidades médicas "de hombres". EFEsalud. 08 de marzo de 2019. Disponible en: https://www.efesalud.com/mujeres-pioneras-especialidadesmedicina 
3. Fernández Gámez N. En Traumatología todo el instrumental médico está pensado para hombres. La Revista. 16 de enero de 2019. Disponible en: https://www.redaccionmedica.com/ la-revista/noticias/-en-traumatologia-todo-el-instrumentalmedico-esta-pensado-para-hombres--7612

4. Rico-Godoy C. Como ser una mujer y no morir en el intento. Barcelona: Ediciones Temas de Hoy; 1990.

5. Corona Vázquez T, Medina Mora ME, Ostrosky Wegman P, Sarti Gutiérrez EJ, Uribe Zúñiga $P$ (eds.). Academia Nacional de Medicina/México. La mujer y la salud en México. México: CONACYT; 2014.

6. Savvidou OD, Zampeli F, Antoniadou T, Van Beek A, Papagelopoulos PJ. Pioneer female orthopaedic surgeons as role models. Orthopedics. 2020; 43 (1): e8-e14.

7. Pozo Felguera G. La primera mujer especialista en Traumatología y Cirugía de Granada. El Independiente de Granada. 29 de diciembre de 2019. Disponible en: https:// www.elindependientedegranada.es/ciudadania/primera-mujerespecialista-traumatologia-cirugia-granada

8. Arenas Serna G. Profesores de la ortopedia mexicana, Dra. Hayde Esparza Guevara. Ortho-tips. 2014; 10 (2): 76-78.

9. Castillo Yáñez L. Número de médicas especialistas en traumatología es muy bajo. Saludario. 17 de enero de 2021. Disponible en: https://www.saludiario.com/es-muy-reducidoel-numero-de-las-medicas-especialistas-en-traumatologia/

10. Green JA, Chye PC, Hiemstra LA, Fellander-Tsai L, Incoll I, Weber K, et al. Diversity: women in orthopaedic surgery - a perspective from the International Orthopaedic Diversity Alliance. JTO. 2020; 8 (1): 44-51.

11. International Orthopaedic Diversity Alliance. Diversity in orthopaedics and traumatology: a global perspective. EFORT Open Rev. 2020; 5 (10): 743-752. doi: 10.1302/20585241.5.200022.

12. Gautam M. Women in Medicine: stresses and solutions. West J Med. 2001; 147: 34-41. doi: 10.1136/ewjm.174.1.37.

13. Leblond V, Hatermann A, Autran B. Les femmes médecins aujourd'hui: l'avenir de la médecine? Les Tribunes de la santé. 2014; 3 (44): 43-49. doi: 10.3917/seve.044.0043.

14. Organización Médica Colegial de España. Demografía médica. Apuntes para la eficiencia del Sistema Sanitario. Feminización y especialidades. 2017. Disponible en: https://www.cgcom.es/ sites/default/ files/estudio_demografia_2017/35/\#zoom=z

15. Organización Médica Colegial de España. Diagnóstico de género de la profesión médica. Cuadernos CGCOM. 2018.

16. Sanitaria. 16 de marzo de 2021. Núm. 134. Disponible en: http://sanidadprivada.publicacionmedica.com/noticia/ser-buentraumatologo-no-depende-del-sexo-sino-de-la-ilusion-quepongas-en-lo-que-haces

17. Valdivieso C. 8-M en Sevilla: las mujeres traumatólogas pisan fuerte en el Macarena. Diario de Sevilla. 08 de marzo de 2021.

18. Heinze-Martín G, Olmedo-Canchola VH, Bazán-Miranda G, Bernard-Fuentes NA, Guízar-Sánchez DP. Los médicos especialistas en México. Gac Med Mex. 2018; 154: 342-351. doi: 10.24875/GMM.18003770.

19. Hiemstra LA, Wittman T, Mulpuri K, Vezina C, Kerslake S. Dissecting disparity: improvements towards gender parity in leadership and on the podium within the Canadian Orthopaedic Association. J ISAKOS. 2019; 4: 227-232. doi: 10.1136/ jisakos-2019-000290.

20. Van Heest $A E$, Agel J. The uneven distribution of women in orthopaedic surgery resident training programs in the United States. J Bone Joint Surg Am. 2012; 94 (2): e9. doi: 10.2106/ JBJS.J.01583.
21. Chambers CC, Ihnow SB, Monroe EJ, Suleiman LI. Women in Orthopaedic Surgery: population trends in trainees and practicing surgeons. J Bone Joint Surg Am. 2018; 100 (17): e116. doi: 10.2106/JBJS.17.01291.

22. Day CS, Lage EL, Ahn CS. Diversity based on race, ethnicity, and sex between academic orthopaedic srgery and other specialties. A comparative study. J Bone Joint Surg Am. 2010; 92 (13): 2328-2335. doi: 10.2106/JBJS.I.01482.

23. Rhode RS, Wolf JM, Adams JE. Where are the women in Orthopaedic Surgery? Clin Orthop Relat Res. 2016; 474: 1950-1956. doi: 10.1007/s11999-016-4827-y.

24. Van Heest AE, Agel J, Samora JB. A 15-year report on the uneven distribution of women in Orthopaedic Surgery residency training programs in the United States. JBJS Open Access. 2021; 6 (2): e20.00157. doi: 10.2106/JBJS.OA.20.00157.

25. Bennett CL, Baker O, Rangel EL, Marsh RH. The gender gap in surgical residencies. JAMA Surg. 2020; 155: 893-894. doi: 10.1001/jamasurg.2020.2171.

26. Acuña AJ, Sato EH, Jella TK, Samuel LT, Jeong SH, Chen AF, et al. How long will it take to reach gender parity in Orthopaedic Surgery in the United States? An analysis of the National Provider Identifier Registry. Clin Orthop Relat Res. 2021; 479 (6): 1179-1189. doi: 10.1097/CORR.0000000000001724.

27. Larsen AM, Pories S, Parangi S, Robertson FC. Barriers to pursuing a career in surgery: an institutional survey of Harvard Medican School students. Ann Surg. 2021; 273 (6): 1120-1126. doi: 10.1097/SLA.0000000000003618.

28. Hill JF, Yule A, Zurakowski D, Day CS. Residents' perceptions of sex diversity in orthopaedic surgery. J Bone Joint Surg Am. 2013; 95 (19): e1441-e1446. doi: 10.2106/JBJS.L.00666.

29. Cannada LK, Teuscher DD. Women in orthopaedics: the attraction is mutual. AAOS Now. August 2016. pp. 1-5.

30. Sobel AD, Cox RM, Ashinsky B, Eberson CP, Mulcahey MK. Analysis of factors related to the sex diversity of orthopaedic residency programs in the United States. J Bone Joint Surg Am. 2018; 100 (11): e79. doi: 10.2106/JBJS.17.01202.

31. Helitzer DL, Newbill SL, Cardinali G, Morahan PS, Chang S, Magrane D. Changing the culture of academic medicine: critical mass or critical actors? J Womens Health. 2017; 26 (5): 540548. doi: 10.1089/jwh.2016.6019.

32. O'Connor MI. Medical school experiences shape women student's interest in Orthopaedic Surgery. Clin Orthop Relat Res. 2016; 474: 1967-1972. doi: 10.1007/s11999-016-4830-3.

33. Forriol F. La mujer en la Cirugía Ortopédica y Traumatología. Rev Ortop Traumatol. 2004; 48: 255.

34. Cannada LK. Women in orthopaedic fellowships: what is their match rate, and what specialties do they choose? Clin Orthop Relat Res. 2016; 474: 1957-1961. doi: 10.1007/s11999-0164829-9.

35. Yeo HL, Dolan PT, Mao J, Sosa JA. Association of demographic and program factors with American Board of Surgery qualifying and certifying examinations pass rates. JAMA Surg. 2020; 155 (1): 22-30. doi: 10.1001/jamasurg.2019.4081.

36. Flores-Domínguez C, Meraz Ávila D, Harari DNB. La mujer en la medicina del siglo XXI. Educ Med. 2019; 20 (5): 325-328. doi: 10.1016/j.edumed.2018.03.018.

37. Bucknall B, Pynsent PB. Sex and the orthopaedic surgeon: a survey of patient, medical student and male orthopaedic surgeon attitudes towards female orthopaedic surgeons. Surgeon. 2009; 7 (2): 89-95. doi: 10.1016/s1479-666x(09)80023-1.

38. Pingleton SK, Jones EV, Rosolowski TA, Zimmerman MK. Silent bias: challenges, obstacles, and strategies for leadership development in academic medicine - Lessons 
from oral histories of women professors at the University of Kansas. Acad Med. 2016; 91 (8): 1151-1157. doi: 10.1097/ ACM. 0000000000001125 .

39. Dineen HA, Patterson JM, Eskildsen SM, Gan ZS, Li Q, Patterson BC, et al. Gender preferences of patients when selecting orthopaedic providers. lowa Orthop J. 2019; 39 (1): 203-210.

40. Bates C, Gordon L, Travis E, Chatterjee A, Chaudron L, Fivush $B$, et al. Striving for gender equity in academic medicine careers: A call to action. Acad Med. 2016; 91 (8): 1050-1052. doi: 10.1097/ACM.0000000000001283.

41. Beebe KS, Krell ES, Rynecki ND, Ippolito JA. The effect of sex on orthopaedic surgeon income. J Bone Joint Surg Am. 2019; 101 (17): e87. doi: 10.2106/JBJS.18.01247.

42. Hill JF, Johnson AH, Cannada L. A profile of female academic orthopaedic surgeons. Curr Orthop Pract. 2013; 24 (6): 636640. doi: $10.1097 / B C 0.0000000000000034$.

43. Hiller KP, Boulos A, Tran MM, Cruz Al Jr. What are the rates and trends of women authors in three high-impact orthopaedic journals from 2006-2017? Clin Orthop Relat Res. 2020; 478 (7): 1553-1560. doi: 10.1097/CORR.0000000000001043.

44. Loder RT, Kacena MA, Ogbemudia B, Ngwe HN, Aasar A, Ninad $\mathrm{N}$, et al. Bibliometric analysis of the english musculoskeletal literature over the last 30 years. ScientificWorldJournal. 2021; 2021: 5548481. doi: 10.1155/2021/5548481.

45. Rynecki ND, Krell ES, Potter JS, Ranpura A, Beebe KS. How well represented are women orthopaedic surgeons and residents on major orthopaedic editorial boards and publications? Clin Orthop Relat Res. 2020; 478 (7): 1563-1568. doi: 10.1097/CORR.0000000000000824.

46. Potter JS, Ranpura A, Rynecki ND, Beebe KS, Galdi B. Gender parity in academic leadership roles at AOSSM Annual Meetings. Orthop J Sports Med. 2021; 9 (1): 2325967120979995. doi: 10.1177/2325967120979995.

47. Bratescu RA, Gardner SS, Jones JM, Siff TE, Lambert BS, Harris JD, et al. Which subspecialties do female orthopaedic surgeons choose and why?: identifying the role of mentorship and additional factors in subspecialty choice. J Am Acad Orthop Surg Glob Res Rev. 2020; 4 (1): e19.00140. doi: 10.5435/ JAAOSGlobal-D-19-00140.

48. Klyce W, Nhan DT, Dunham AM, El Dafrawy MH, Shannon C, LaPorte DM. The times, they are a-changing: Women entering academic Orthopedics today are choosing nonpediatric fellowships at a growing rate. J Surg Educ. 2020; 77 (3): 564571. doi: 10.1016/j.jsurg.2019.12.007.

49. Uzoigwe CE, Middleton RG. Occupational radiation exposure and pregnancy in orthopaedics. J Bone Joint Surg Br. 2012; 94 (1): 23-27. doi: 10.1302/0301-620X.94B1.27689.
50. Downes J, Rauk PN, Vanheest AE. Occupational hazards for pregnant or lactating women in the orthopaedic operating room. J Am Acad Orthop Surg. 2014; 22 (5): 326-332. doi: 10.5435/ JAAOS-22-05-326.

51. Hamilton AR, Tyson MD, Braga JA, Lerner LB. Childbearing and pregnancy characteristics of female orthopedic surgeons. J Bone Joint Surg Am. 2012; 94 (11): e77. doi: 10.2106/ JBJS.K.00707.

52. Rochon PA, Davidoff F, Levinson W. Women in academic medicine leadership: has anything changed in 25 years? Acad Med. 2016; 91 (8): 1053-1056. doi: 10.1097/ ACM.0000000000001281.

53. Errani C, Tsukamoto S, Kido A, Yoneda A, Bondi A, Zora F, et al. Women and men in orthopaedics. SICOT J. 2021; 7: 20. doi: $10.1051 /$ sicotj/2021020.

54. Xepoleas MD, Munabi NC, Auslander A, Magee WP, Yao $\mathrm{CA}$. The experiences of female surgeons around the world: a scoping review. Hum Resour Health. 2020; 18 (1): 80. doi: 10.1186/s12960-020-00526-3.

55. Emery SE; Carousel Presidents. Diversity in orthopaedic surgery: international perspectives: AOA critical issues. J Bone Joint Surg Am. 2019. doi: 10.2106/JBJS.18.00355.

56. International Orthopaedic Diversity Alliance. Available in: https://www.orthopaedicdiversity.org

57. The Perry Initiative. Available in: https://www.perryinitiative.org

58. Nth Dimensions. Available in: https://www.nthdimensions.org

59. Boletín Oficial del Estado. Reino de España. Ley Orgánica $3 / 2007$, de 22 de marzo, para la igualdad efectiva de mujeres y hombres. BOE núm. 71 de 23 de marzo de 2007.

60. Casado-Mejía R, Botello-Hermosa A. Representatividad de las mujeres en las sociedades científicas españolas del ámbito de la salud en 2014. Gac Sanit. 2015; 29 (3): 209-212. doi: 10.1016/j.gaceta.2014.09.010

61. Botello-Hermosa A, Casado-Mejía R, Germán-Bes C. Presencia de las mujeres en los órganos de dirección de los colegios profesionales del ámbito de la salud en 2015. Rev Esp Salud Publica. 2015; 89 (6): 627-632. doi: 10.4321/S113557272015000600010.

62. Pastor Gosálbez MI, Belzunegui Eraso A, Pontón Merino P. Mujeres en sanidad: entre la igualdad y la desigualdad. Cuadernos de Relaciones Laborales. 2012; 30 (2): 497-518. doi: 10.5209/rev.CRLA.2012.v30.n2.40210.

\section{Conflicto de intereses}

Ninguno.

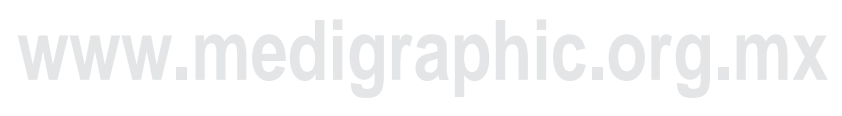

